\title{
Cloud Computing: Comparison with Previous Technique and Research Challenges
}

\author{
Nimisha Gupta \\ B.tech (IT), \\ N.I.E.C, GGSIPU \\ New Delhi, India
}

\author{
Bharti Chauhan \\ B.tech (IT), \\ N.I.E.C, GGSIPU \\ New Delhi, India
}

\author{
Tanvi Anand \\ B.tech (IT), \\ N.I.E.C, GGSIPU \\ New Delhi, India
}

\author{
Charul Dewan \\ Assistant \\ Professor(IT), \\ N.I.E.C, GGSIPU \\ New Delhi, India
}

\begin{abstract}
Cloud computing has recently emerged as a new paradigm for hosting and delivering the services over the Internet. It is an enhanced version of client-server application. In this paper, we will present comparison between cloud computing and clientserver application, highlighting key concepts of cloud computing, its architectural principles, as well as research challenges and its environmental impact. The aim of this paper is to provide better understanding of need of cloud computing. [6]
\end{abstract}

\section{General Terms}

Client server architecture, cloud computing, characteristics and environmental impact of cloud

\section{INTRODUCTION}

Cloud computing, a rapidly developing information technology, has aroused the concern of the whole world. With the increasing demand of IT companies for processing large amount of data and better storage. Cloud computing has emerged as a promising approach to rent a large IT infrastructure on a short-term pay per usage basis. With this rapid development in technology will lead to recognition of a new computing model called cloud computing, in which resources are provided as general utilities that are used by user through internet.

Earlier Client-server application model was used which is a distributed application structure that partition tasks or workload between the providers of resource called server and service requester called client. Server programs share their resources with client but client doesn't, it can only request for server content. A client is a computer or computer program that initiates contact with server in order to use resources.

Problems in the client server application are: dependability (when the server goes down the operation cease), lack of scalability, higher cost and occurrence of network congestion.

Thus the, beginning of cloud computing has made a enormous impact on the IT industry, where large companies such as Google made an effort in providing more powerful, reliable and cost efficient cloud platform. Also, cloud computing provide many features that attract the business owner towards this new paradigm, shown below:

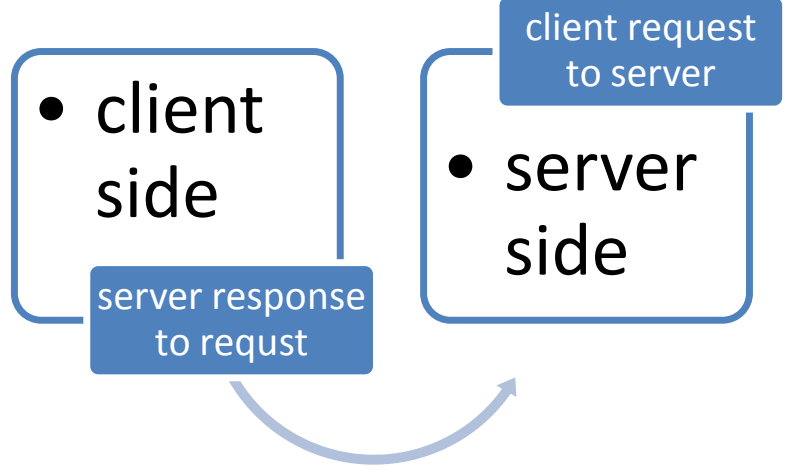

Fig 1: client server model

a. No up-front investment: A service provider does not need to invest for gaining benefits of cloud computing. It simply rent resources from cloud according to its need and pay for its usage.

b. Lowering operating cost: In cloud, resources are allocated and de-allocated rapidly on demand, therefore providing saving of operating cost.

c. High measurability: It allows the service provider to push large amount of sufficient data in the cloud and make them easily accessible. A service provider can easily expand its service to large scale so as to fulfill the increasing demand of resources. This method is sometimes calls surge computing. [1]

d. Easily accessible: Resources hosted on cloud are web based. Therefore, easily accessible by any devices having internet connection.

e. Increased ability to handle business risk: In cloud, infrastructure providers are better fitted for handling risk.

\section{OVERVIEW OF CLOUD COMPUTING}

In this section, a brief overview about the cloud computing has being given, including definition and comparison with other technique.

\subsection{Definition}

Cloud computing was introduced in 1960s by John McCarthy. He was the first one who gave the idea that computing facility will be provided to the public like utility [39]. The term "cloud" in telecommunication means a public or semi public space on transmission lines that exist at the end points of transmission in 1990s. The term "cloud computing" is a 
expression that describes a variety of computing concepts that involve large number of computer connected to a real time communication network such as internet. The word cloud computing is introduced in 2006 by large companies such as Google, Amazon to describe the new paradigm in which the people are increasingly accessing software, files over the web.

The term cloud computing based on marketing to represent various ideas, but the lack of standard definition of cloud computing has generated not only market hypes but also leads to a lot of confusion. The standard definition of cloud computing given by National Institute of Standard and Technology (NSIT) [6]. Definition given by NSIT: cloud computing is a model for enabling ubiquitous, convenient, ondemand network access to a shared pool of configurable computing resources (e.g., networks, servers, storage, applications, and services) that can be rapidly provisioned and released with minimal management effort or service provider interaction. Cloud computing comes into focus only when we think about what IT always needs: a way to increase capacity or add capabilities on the fly without investing in new infrastructure, training new personnel, or licensing new software. Cloud computing include pay as we use service over the Internet.Cloud computing is a type of computing that relies on sharing computing resources rather than local server or personal devices to handle application.

\section{DIFFERENCE BETWEEN CLIENT SERVER ARCHITECTURE AND CLOUD COMPUTING}

- Cloud computing is a kind of environment where our software/hardware can be hosted.

- In cloud computing we can host ' $\mathrm{N}$ ' number client server kind of applications.

- The major difference between client server and cloud computing is that, the server is usually local. The employees access it over a private network. It is owned and operated by the employer and used exclusively by the employees. Cloud computing is the server accessed through the internet. The servers are owned by big companies like Google that run applications and many start-ups that provide data storage.

- "Cloud computing "is the latest technology but its actual meaning varies. When people say cloud computing they are mostly referring to storing their data "online". That is we are storing our data at another location usually managed by a third party. But the disadvantage is that it requires constant internet connection.

\section{CLOUD COMPUTER ARCHITECTURE}

This portion includes architectural, business and various operational models of cloud computing.[3]

\subsection{Architectural model}

This model comprises of 3 layers:

a. Infrastructure layer: Also known as the virtualization layer [2], the infrastructure layer creates a pool of storage and computing resources by partitioning the physical resources using virtualization technologies such as XEN, KVM, etc . It also manages the physical resources of the cloud like servers, routers, switches, power and cooling system. All these components are implemented in data centers. The infrastructure layer is anessential component of cloud computing, since many keyfeatures, such as dynamic resource assignment, are onlymade available through virtualization technologies.

b. Platform layer: It exists on the top of the infrastructure layer, and comprises of operating systems and application frameworks. The purpose of this layer is to minimize the burden of placing applications directly into VM containers. For example, Google App Engine operates at the platform layer to provide API support for implementing storage, database and business logic of typical web applications.

c. Application layer: The application layer is the ultimate goal of cloud computing and the most common from a user perspective. For example, a user can access data stored in a database in U.S.A via a mobile phone in Australia or watch a video located on a server in China from his laptop in the U.S. The Internet is itself an information cloud.

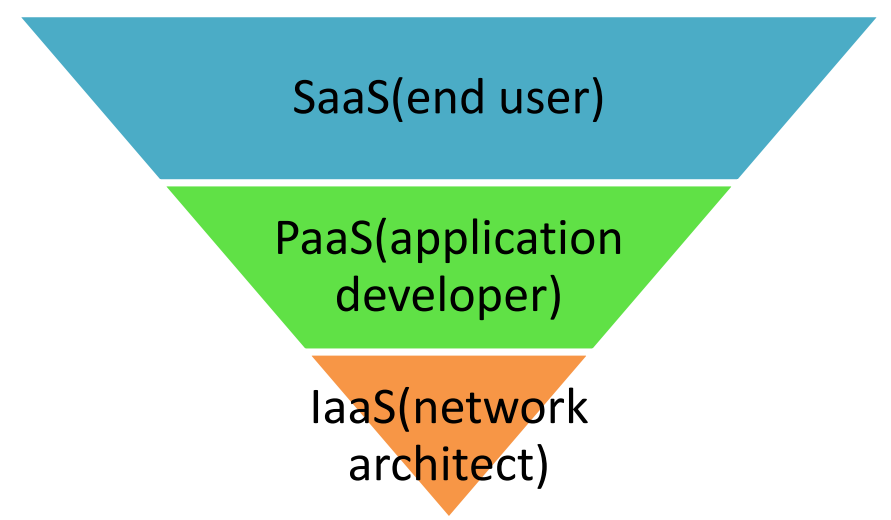

Fig 2: layered architecture of cloud computing

\subsection{Business model}

Business Process as a Service (BPaaS) is any type of horizontal or vertical business process that's delivered according to the cloudservices model. These cloud services include Software as a Service (SaaS), Platform as a Service (PaaS), and Infrastructure as a Service (IaaS).

There is a practical approach in selecting a business process service. Firstly, an organization can select a process that is suitable to their business policy. This ensures that a welldefined and a consistent process exists in the organization. For example, a company may have a complex process for managing transactions. This service can be associated with other services in the cloud as well as to the applications in the data center.

In this figure we showed 3 business services: 


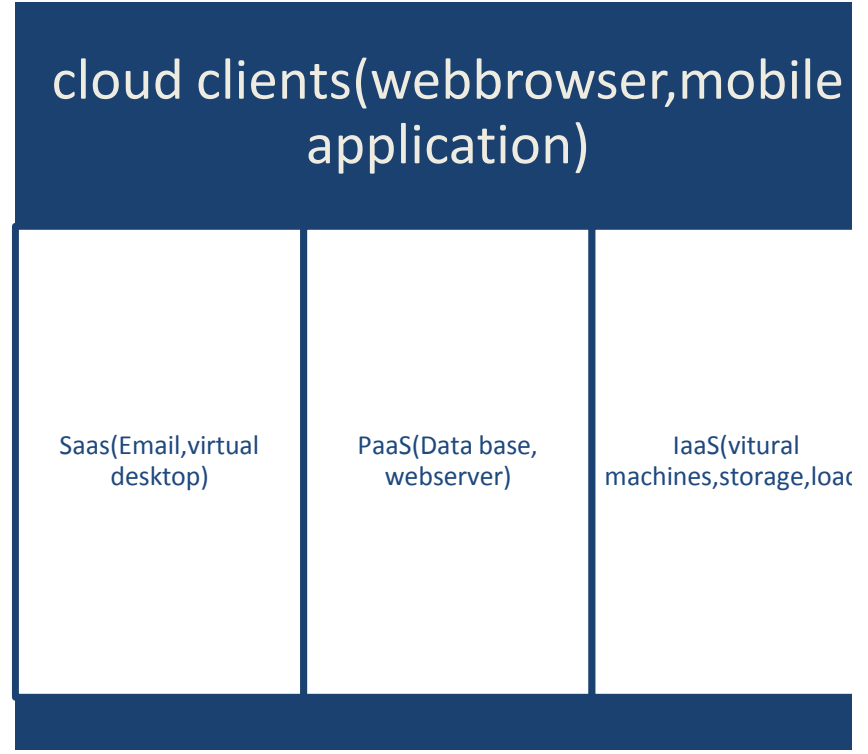

Fig 3: Business model of cloud computing

a. Infrastructure as a Service (IaaS): It is a model that provides equipment for supporting different operations like storage, hardware, servers and networking components. The service provider is the owner of the equipments and has the maintenance responsibility. The client pays as per the usage. For ex: Amazon EC2.

b. Platform as a Service (PaaS): In this the hardware, operating systems, storage and network capacity are rented over the Internet. It allows the customers to use virtualized servers and corresponding services for executing existing applications or creating and testing new ones. For ex: Google App Engine.

c. Software as a service (SaaS):It is a software distribution model in which applications are hosted by service provider and is available to customers over the Internet For ex: Salesforce.com.

Client server architecture can also be used in business based model but by using cloud computing we can efficiently access the data. It is efficient in terms of saving energy.

\subsection{TYPES OF CLOUDS}

With cloud computing technology, large number of resources can be linked through private or public networks. This technology modifies infrastructure planning and provides dynamically measurable infrastructure for cloud related applications, data, and file storage.

a. Public cloud: Public clouds are available to the general public through a service provider who hosts the cloud infrastructure. Public cloud providers like Amazon AWS, Microsoft and Google are the owners and operate the infrastructure and provide access over the network. In this model, customers have no control over the located infrastructure.

b. Private cloud: It is dedicated to a particular organization. It also allows businesses to host applications in the cloud, while locating concerns regarding data security and control, which is often lacking in a public cloud environment. It is not shared with other organizations, but managed by a thirdparty.

Client server architecture resembles the private cloud in many ways as it is also dedicated to a particular organization but differs in the way that private clouds implements the concept of virtualization.

c. Hybrid cloud: This cloud consists of two or more clouds (private, community or public) that are linked together offering the advantages of multiple deployment models. In this, we can take advantage of third party cloud providers in either a full or partial manner; increasing the flexibility of computing. By replacing a private cloud with the resources of a public cloud we can manage any unexpected surges in workload.

d. Community cloud:It is a multi-tenant cloud service model that is shared among various organizations and that is maintained and secured by all the participating organizations. This is a hybrid form of private clouds built and operated specifically for a targeted group which communities have similar cloud requirements and their ultimate goal is to work together to achieve their business objectives. The goal of community clouds is to have participating organizations realize the benefits of a public cloud with the added level of privacy, security, and compliance usually associated with a private cloud.

e. Virtual private cloud: It is on demand configurable pool of shared computing resources allocated within a publiccloud environment, providing certain level of isolation between the different organizations. The isolation between one VPC user and all other users of the same cloud (other VPC users as well as other public cloud users) is achieved normally through allocation of a Private IP Subnet and a virtual communication construct (such as a VLAN or a set of encrypted communication channels) per user. In a VPC solution, the previously described mechanism, providing isolation within the cloud, is accompanied with a VPN function (again, allocated per VPC user) that secures, by means of authentication and encryption, the remote access of the organization to its VPC cloud resources. With the introduction of the described isolation levels, an organization using this service is in effect working on a 'virtually private cloud (i.e. as if the cloud infrastructure is not shared with other organizations).

\section{CLOUD COMPUTING CHARACTERSTICS}

a. On-demand capabilities: A business will secure cloud-hosting services through a cloud host provider which could be our usual software vendor. We have access to these services and have the power to change cloud services through an online control panel or directly with the provider. We can add or delete users and change storage networks and software as needed. Typically, we have to pay-for-what-we-use scenario. Terms of subscriptions and payments will vary with each software provider. [1] 
b. Broad network access: Our team can access business management solutions using their smart phones, tablets, laptops, and office computers. They can use these devices wherever they are located with a simple online access point. This mobility is particularly attractive for businesses so that during business hours or on off-times, employees can stay on top of projects, contracts, and customers whether they are on the road or in the office. Broad network access includes private clouds that operate within a company's firewall, public clouds, or a hybrid deployment.

c. Resource pooling: The cloud enables your employees to enter and use data within the business management software hosted in the cloud at the same time, from any location, and at any time. This is an attractive feature for multiple business offices and field service or sales teams that are usually outside the office

d. Rapid elasticity: The cloud is flexible and scalable to suit our immediate business needs. We can quickly and easily add or remove users, software features, and other resources.

e. Measured service: Going back to the affordable nature of the cloud, we only pay for what you use. We and our cloud provider can measure storage levels, processing, bandwidth, and the number of user accounts and we are billed appropriately. The amount of resources that you may use can be monitored and controlled from both our side and cloud provider's side provides an excellent feature of transparency.[1]

\section{CHALLENGES FACED BY \\ COMPANIES THAT WANT TO MOVETO A PUBLIC CLOUD [4]}

a. Security: With cloud computing, we can heavily dependent on the service provider for security. Cloud service providers can claim to provide complete security for access, compliance, data segregation, backup, recovery, etc. [2]

b. Data Ownership: What happens to our data when it leaves our organization to reside in the cloud? Companies who move to the cloud probably will not completely lose track of their data but they are likely to lose some level of ownership and control. It is important to understand who can access the data and for what purpose.

c. Lock-in and Interoperability: Now days each service offering provides its own unique way for the cloud to interact with applications, data and clients. It can be very difficult to use multiple vendors and to have consistent cloud services.

d. Standard Architecture: There is no standard open architecture being used for cloud services. Each of the major cloud providers (Amazon Web Services, Salesforce Force, Google App Engine and Microsoft Azure) imposes different architectures that are dissimilar to the common architectures currently used for enterprise apps. Although a customer's functional and technical requirements may be satisfied, the lack of standards will compromise the customer's ability to consistently migrate from one service provider to another and may need a complete rewrite of their software to do so. e. Enterprise Support and Service Maturity: Cloud computing services may not provide the levels of reliability, manageability, and support required by large enterprises.

f. Loss of data: Data stored in the cloud can be replicated across multiple machines and backed up. However, not all cloud services have the same redundancy for disaster recovery.

g. Return on Investment: The expectation is that external cloud computing can reduce costs. However, the cost advantage for large enterprises may not be as clear as for SMEs. Currently, many large enterprises can reap the benefits of significant economies of scale in their own internal IT operations.

h. Requirement for online connectivity: Cloud computing is impossible if you cannot connect to the internet. A dead internet connection means it is not possible to work, and in areas where internet connections are few or inherently unreliable, this could be a problem.

\section{HOW DOES CLOUD COMPUTING REDUCE THE ENVIRONMENTAL IMPACT OF IT[5]}

To understand the potential advantage of cloud computing in more detail, it is important to look at the distinct factors contributing to a lower per-user carbon footprint. These factors apply across cloud providers in general and are even relevant for many on-premise scenarios. This level of understanding can thus help IT executives target additional efficiency gains in an on-premise environment and realize additional performance advantages in the future. Generally speaking, the comparatively smaller carbon footprint of cloud computing is a consequence of both improved infrastructure efficiency and a reduced need for IT infrastructure to support a given user base. In turn, these primary levers are heavily influenced by four key factors:

- Dynamic Provisioning

- Multi-Tenancy

- Server Utilization

- Data Center Efficiency

a. Dynamic Provisioning

IT managers typically deploy far more server, networking and storage infrastructure than is actually needed to meet application demand. Over-provisioning is certainly understandable business demand for services exceeds what IT can provide. Thus infrastructure planning is typically conducted with a conservative, "just in case" mindset that results in capacity allocation that is not aligned with actual demand. By contrast, cloud providers tend to manage capacity much more diligently, because over-provisioning at the cloud's operational scale can be very expensive. Providers typically have dedicated resources to monitor and predict demand and continually adjust capacity, and their teams have developed greater expertise in demand modeling and in the use of sophisticated tools to manage the number of running servers. Thus, cloud providers can reduce the inefficiency caused by over-provisioning by optimizing the number of active servers to support a given user base.

b. Multi-Tenancy

Just as multiple tenants in an apartment building use less power overall than the same number of people owning their own homes, so do the multiple tenants of a cloud-provided infrastructure reduce their overall energy use and associated 
carbon emissions. The cloud architecture allows providers to simultaneously serve multiple companies on the same server infrastructure. Different demand patterns from numerous companies reduce overall demand peaks and make fluctuations more predictable. The ratio between peak and average loads becomes smaller, and that in turn reduces the need for extra infrastructure. Major cloud providers are able to serve millions of users at thousands of companies simultaneously on one massive shared infrastructure. By operating multi-tenant environments, cloud providers can reduce overhead for onboarding and managing individual organizations and users. The Microsoft cloud offerings analyzed in this study are relatively new and are currently experiencing rapid growth. The more mature a given cloud service becomes, the less the demand will fluctuate, resulting in even greater energy savings in the future.

\section{c. Server Utilization}

Cloud computing can drive energy savings by improving server utilization (the measurement of the portion of a server's capacity that an application actively uses). As large-scale cloud providers tend to run their infrastructure at higher and more stable utilization levels than corresponding on-premise operations, the same tasks can be performed with far fewer servers. Whereas a typical on-premise application may run at 5 to 10 percent average utilization rate, the same application in the cloud may attain 40 to 70 percent utilization, thus dramatically increasing the number of users served per machine. It is important to note that while servers running at higher utilization rates consume more power, this results in increase of relative performance gains. Virtualization offers a strategy to improve server utilization for both cloud and onpremise scenarios by allowing applications to run in an environment separated from the underlying physical servers. IT organizations can measure individual virtual resources to fit application needs instead of allocating an entire physical system whose full capability is not utilized. In this way, virtualization provides a tool for IT departments to narrow the efficiency gap between on-premise deployment and a multitenant cloud service.

\section{Data Center Efficiency}

Data center is designed in the way facilities are physically constructed, equipped with IT and supporting infrastructure impact on the energy use for a given amount of computing power. A common measure of how efficiently a data center uses its power is called power usage effectiveness ratio (PUE). Power usage effectiveness is defined as the ratio of overall power drawn by the data center facility to the power delivered to IT hardware. For example, a power usage effectiveness of 1.5 means that for every $1 \mathrm{kWh}$ of energy consumed by IT hardware, the data center must draw $1.5 \mathrm{kWh}$ of energy, with $0.5 \mathrm{kWh}$ used for cooling of IT equipment. Standardizing and measuring average power usage effectively across companies can be difficult.. Today's data center designs for large cloud service providers achieve power usage should be as low as 1.1 to 1.2. This efficiency gain could reduce power consumption over traditional enterprise data centers by 40 percent through data center design alone. Innovations like modular container design, cooling that relies on outside air or water evaporation, or advanced power management through power supply optimization, are all approaches that have significantly improved power usage effectiveness in data centers. As cloud computing gains larger adoption and the share of data processing performed by modern data center facilities increases, the industry's PUE averages should improve. These two trends will drive greater efficiency in data centers.

\section{CONCLUSION}

In this paper, we discussed about the client server model and drawbacks in this model which leads to the shift technology from client server application to cloud computing as client server application only involves one to one communication but in cloud computing we access data from anywhere and anytime.

Cloud computing has recently emerged as a compelling paradigm for managing and delivering services over the Internet. The rose of cloud computing is rapidly changing the picture of information technology, and leads to the turning of obtaining the promise of utility computing into a reality. However, the lack of significant benefits offered by cloud computing, the current technologies are not matured enough to realize its full potential. Many important challenges in this domain, including automatic resource provisioning, power management and security management, are starting to receive attention from the research community. Therefore, we believe there is a opportunity for researchers to have a tremendous beginning that never happened, and bring significant impact to their development in the industry.

\section{REFERENCES}

[1] Shufen zhang, shaui zhsng, xue bin chen, shang zhou wu, "Analysis and research of cloud computing instance". Published on January 2010.

[2] Wentao liu, "research on cloud computing security,problem and strategy". Published on April 2012

[3] Pankaj arora,Rubal chaudhary Wadhawan, Satinder pal ahuja " cloud computing security issues in infrastructure as a service". Published on January 2012.

[4] Slyas Syoob,Emrab Zarifogn, A.B. Dicker "cloud computing operations research".

[5] Jayant Balinga,Robert W.A Ayre, Kerry Hinton and Rodney S.Tucker "Green cloud computing: Balancing energy in processing storage and transport". Published on January 2011

[6] Qi Zhang, Lu Cheng, Raouf Boutaba Cloud computing: "State of art and research challenges. Published on April 2010. 